\title{
Amplified spontaneous emission based quantum random number generator
}

\author{
Ádám Marosits ${ }^{1}$, Ágoston Schranz ${ }^{2}$, and Eszter Udvary³
}

\begin{abstract}
There is an increasing need for true random bits, for which true random number generators (TRNG) are absolutely necessary, because the output of pseudo random number generators is deterministically calculated from the previous states. We introduce our quantum number generator (QRNG) based on amplified spontaneous emission (ASE), a truly random quantum physical process. The experimental setup utilizes the randomness of the process. In this system, optical amplifiers (based on ASE) play the major role. The suitable sampling rate is selected in order to build the fastest generator, while avoiding the correlation between consecutive bits. Furthermore, the applied post-processing increases the quality of the random bits. As a results of this, our system generated random bits which successfully passed the NIST tests. Our real-time generation system - which is currently a trial version implemented with cheap equipment - will be available for public use, generating real time random bits using a web page.
\end{abstract}

Index Terms - quantum random number generator, amplified spontaneous emission, sampling rate, real-time generation

\section{INTRODUCTION}

Nowadays, there is an ever increasing demand for random numbers in communication and cryptography. The applications of random numbers include symmetric key cryptography, Monte Carlo simulations, protection of transactions, and key distribution systems, which will be more significant in the age of quantum computers. In order to generate true random bits (TRB), quantum random number generators (QRNGs) need to be implemented. Pseudorandom number generators (PRNGs) are widespread; they are cost-efficient because they algorithmically create seemingly random numbers, but they are deterministic, therefore these numbers cannot be declared as truly random. There are some random number generators, which sample complex physical processes, but with suitable measurements others can obtain the same numbers. Nevertheless, the randomness of quantum mechanics can provide high bit generation rates. Some quantum process based generators, for instance the radioactivity based QRNG, come with several serious problems: for example, the radiation is only enough just for a few detections per second, decreasing the generation rate. Moreover, we need huge quantities of radioactive materials, for which serious security arrangements need to be implemented. There are different possible processes for random number generation (e.g. the noise of chaotic circuits or the Brownmotion of particles), but it is not possible to generate high bit generation rates using these phenomena. We can differentiate between optical based QRNG systems, too. The first group is that of is the branching path generators, when the photon goes to a semi-transparent mirror that transmits it along one of the paths. At the end of both paths there is one detector, and the number of the detector signalling the arrival of a photon determines the value of the bit. The semi-

1,2,3 Department of Broadband Infocommunication and Electromagnetic Theory, Budapest University of Technology and Economics, Hungary

1,2 BME Balatonfüred Student Research Group, Budapest University of Technology and Economics, Hungary

1,2,3 E-mail: \{marosits.a, schranz, udvary.eszter\}@hvt.bme.hu transparent mirror is essentially a Hadamard gate, and at the end of the system, the value of the bit is 0 in $50 \%$, and 1 in $50 \%$. The second group are the photon counting generators. In this case, we count photon arrivals in a fixed-length time window, and we can decide on the value of the bit with a predetermined method. The third group is that of the time-of-arrival generators: random bits are generated based on the fluctuation of the time difference between photon arrivals. It is similar in principle to radioactivity based generators, but it is much more secure, since photons are used instead of particle radiation.

There is an another group of QRNGs that utilizes the randomness of amplified spontaneous emission (ASE) in order to generate random bits. The earliest proposal splits the signal into orthogonally polarized components with a polarization splitter, and calculates the difference between the independent polarization components; another one uses a balanced power splitter and tunable delay to symmetrize the intensityfluctuation [1]. In order to not limit the generation rate, some earlier setup operates with optical filters, which have higher bandwidth than the receiver, to avoid its saturation. Some authors digitized the unfiltered, unamplified intensity-fluctuation at 16/32 bits from ASE sources. They got high bit generation rates and reduced the correlations by discarding several MSBs $[2,3]$. Several authors $[2,3,4,5]$ used XOR post-processing methods to reduce short-term correlations. One article mentioned that the signal from a SLED (having a wide quasi-constant spectrum) is split and compared to the reference level to generate random bits [5].

In this paper, we present a QRNG that is based on amplified spontaneous emission. In the following sections, we discuss the theoretical background, the system, the suitable sampling rate selection, the success of post-processing and real-time bit generation. Our experimental setup operates with optical filters (CWDM, providing higher bandwidth than other standards) in order to avoid the saturation in the receiver. Furthermore, the above mentioned XOR post-processing method is applied to reduce short-term correlations. The signal is digitized at 1 bit, so that the quality of randomness will be easily investigated. If we compare the achieved $4 \mathrm{Gbps}$ bit generation rate with the previous setups, we could find several faster implementations (the minimum rate was $2.5 \mathrm{Gbps}$ [1], the maximum was $1.6 \mathrm{Tbps}$ [3]), but digitizing at more bits and discarding several MSBs to reduce correlations, our setup could potentially achieve significantly higher bit generation rates.

\section{THeORETICAL BACKGROUND}

It is necessary to investigate the theoretical background of the phenomena in our setup, so that the generator can work as intended, and problematic operation can be avoided. Here we discuss these aspects in detail.

\section{A. Amplified spontaneous emission}

Optical fiber amplifiers used in optical communications operate based on the effect of stimulated emission [6]. If an atom is in an excited state, it may, after some time, spontaneously decay into a lower energy level, releasing energy in the form of a photon. This process is called 
spontaneous emission [7]. However, it is also possible that the photon emission is stimulated by incoming photons, if these photons have suitable energy. This process is called stimulated emission. In that case the two photons in the output have identical properties. For stimulated emission to dominate over other types of light-matter interaction, population-inversion is required. It means that the population of particles is higher in the upper energy level than in the lower energy level. In many cases, it is achieved by optical pumping. If populationinversion exists, some of the particles from the excited state return spontaneously to the ground state. The photons, that are derived from spontaneous emission, may participate in stimulated emission; therefore, the optical amplifier amplifies its noise, too. The lack of input signal has several advantages: we don't have to filter the deterministic component and the accumulated energy is used to amplify the photons from spontaneous emission. This process is called amplified spontaneous emission (ASE) [8]. The emitted photons have random properties - for instance frequency -, so the amplified sum of the individual electric fields appears at the output as a swiftly fluctuating noise. The parameters of these photons don't correlate with the parameters of the signal photons. ASE cannot be described with classical electrodynamics; it is a quantum physical process. The generator - based on ASE - can generate true random bits using a method, where the measured intensity-fluctuation is compared to the mean, or in our case to the median (above the median a bit " 1 " is assigned to the sample, below the median a " 0 "). The bit generation rate is restricted by the device with the narrowest bandwidth, usually the detector [9].

\section{B. ASE sources}

Several types of devices can be used as ASE sources. In case of semiconductor optical amplifiers (SOA) [10], the population-inversion is achieved by current injection. Without any input signal, the SOA uses the accumulated energy to amplify its own noise originating from spontaneous emission. In this mode, the SOA functions as an ASE source.

Erbium-doped fiber amplifiers (EDFA) [11] are optical fiber amplifiers, where the necessary energy is provided by a laser diode. The pumping laser provides the population inversion. These lasers generally operate at $980 \mathrm{~nm}$ or $1450 \mathrm{~nm}$. After the excitation, there is a quick non-radiative transfer of the ions to a metastable energy level, from where they may return the lower energy level, releasing a photon with a wavelength around $1550 \mathrm{~nm}$ by stimulated emission. In this case we can speak about a quasi-three-level transition [12]. This is an intermediate situation, where the lower energy level is so close to the ground state, because there is an appreciable population in thermal equilibrium at the operating temperature. The particles from the metastable state may return to this state by emitting lower energy photons. This energy loss is called reabsorption loss.

The EDFA in our laboratory was a part of a DWDM infocommunication system, where energy saving is an important aspect; therefore, it doesn't turn on at low input powers $(<-29 \mathrm{dBm})$. Consequently, this equipment is not used as an ASE source, but it provides a high gain, so it is suitable for amplification in our system.

\section{Saturation of the optical-electrical converter}

The lightwave converter is responsible for converting optical intensity to electrical voltage. The device is essentially a photodiode with a transimpedance amplifier (TIA). In our system, the lightwave converter provides the connection between the optical system and the oscilloscope. The photodiode is a photosensitive diode operating based on the photoelectric effect. The incoming photons are absorbed, generate a photocurrent, and this photocurrent is converted to voltage by the TIA. Consequently, the electric voltage is proportional to the optical intensity; more precisely, to the square of the optical field strength. The saturation of the lightwave converter can cause false measurement results and the equipment may be damaged if the incoming power is too high. Saturation happens when the increasing optical power cannot increase the voltage with the same linearity as before. The power-voltage characteristic of our lightwave converter can be seen in Figure 1. The $\mathrm{P}-\mathrm{V}$ characteristic is linear below $5 \mathrm{dBm}$, but above this values it does not increase at the same rate. This is the saturation power; therefore, we have to maximize our system's optical output power under $5 \mathrm{dBm}$.

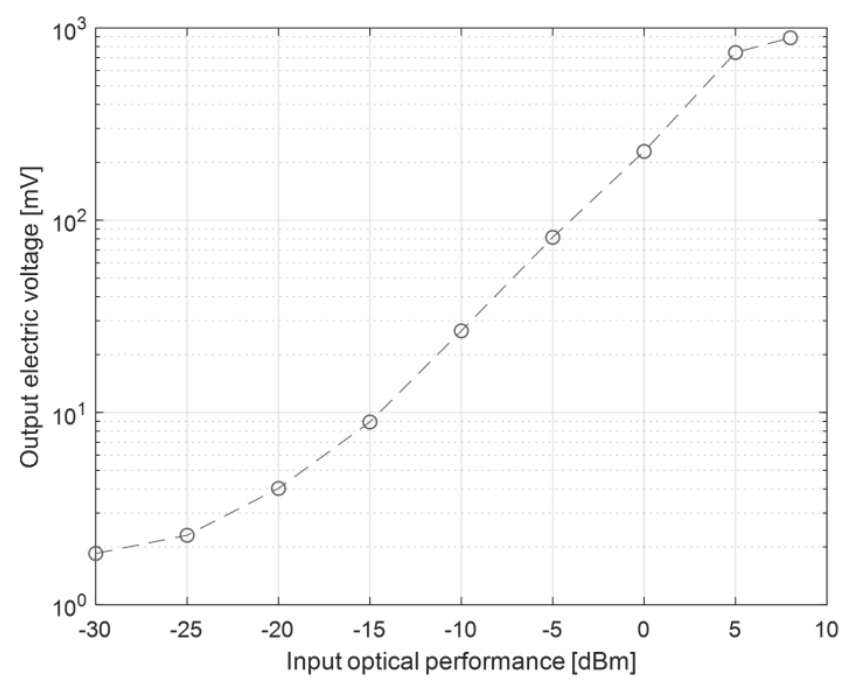

Figure 1. The P-V characteristic of the lightwave converter.

\section{Asymmetric intensity-fluctuation}

The asymmetry of the measured intensity-fluctuation has caused a significant amount of problems during measurements, so the reason behind it and the solution against it need to be clarified. The signal appearing at the output of the lightwave converter can be described as a random variable following a gamma distribution.

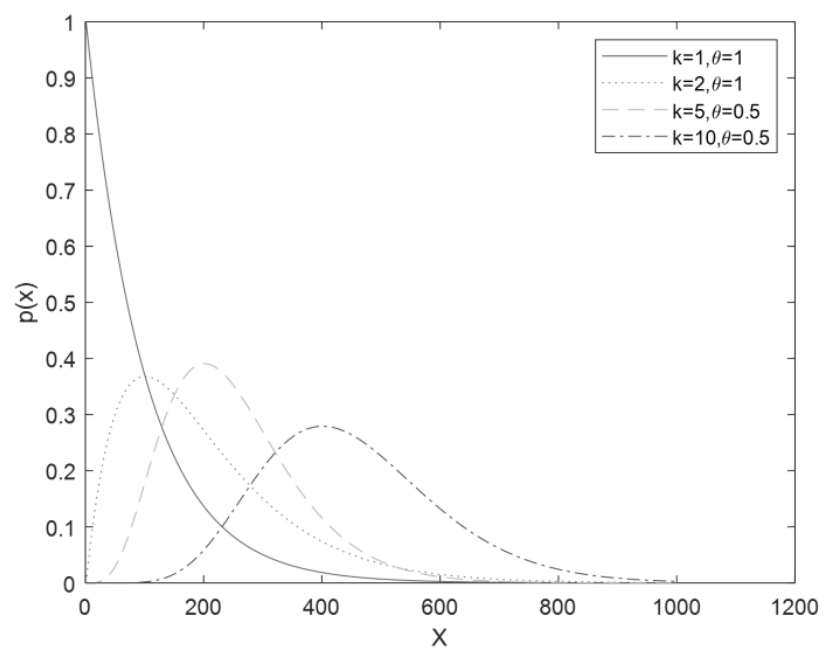

Figure 2. The gamma distribution's probability function with different parameter values for $\mathrm{k}$ and $\Theta$.

The gamma distribution has an asymmetric probability density function (PDF). The PDF (Figure 2.) - using the shape-scale parametrization - can be written as 
Amplified spontaneous emission based

quantum random number generator

$$
f(x, k, \Theta)=\frac{x^{k-1} \cdot e^{-\frac{x}{\Theta}}}{\Theta^{k} \cdot \Gamma(k)},
$$

where $\Gamma(k)$ is the gamma function, $\mathrm{k}$ is the shape parameter, $\theta$ is the scale parameter. For large $\mathrm{k}$, the asymmetric gamma distribution converges to a symmetric normal distribution with mean $\mu=\mathrm{k} \cdot \theta$ and variance $\sigma^{2}=\mathrm{k} \cdot \theta^{2}$. The skewness of the gamma distribution only depends on the shape parameter, and is equal to $2 / \sqrt{k}$ and inversely proportional to the square root of the optical intensity. Consequently, large optical intensities provide quasi-symmetric distributions, which is beneficial, if we would like to achieve a uniform distribution of 0 and 1 bits. The large optical intensity results in a large mean of the intensity-fluctuation. In Figure 3., there are two color scale displays of intensity-fluctuations with different means. The difference between an asymmetric and a quasi-symmetric distribution is clearly visible.
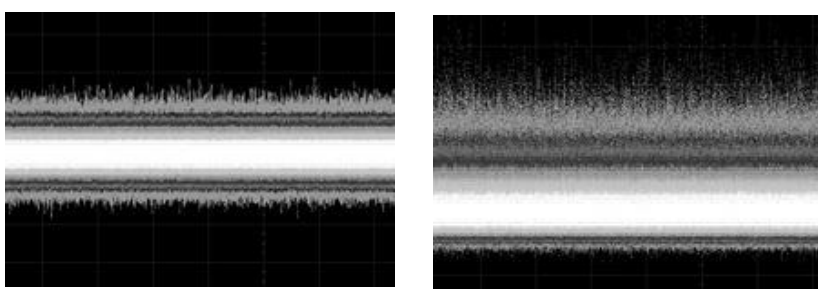

Figure 3. Left: the color scale display of the intensity-fluctuation with high DC voltage $(631 \mathrm{mV})$, right: the color scale display of intensity-fluctuation with low DC voltage $(33 \mathrm{mV})$. The first follows a highly symmetric distribution, while the second is highly asymmetric.

\section{EXPERIMENTAL SETUP}

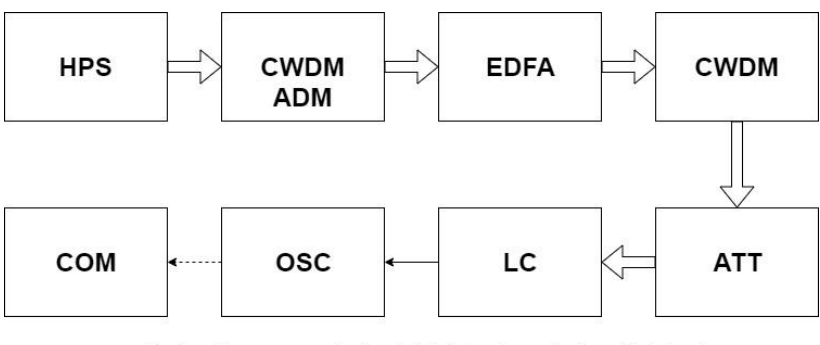

Random bits Analog electrical signal Analog optical signal

Figure 4. The experimental setup's block diagram.

The experimental setup uses a Perkin-Elmer High Power Source (HPS) as the source of ASE. It has a similar spectrum to the SOA, but it has higher noise power near $1550 \mathrm{~nm}$ (the difference is $10 \mathrm{~dB}$ ), where our system operates. The two spectra can be compared in Figure 5 .

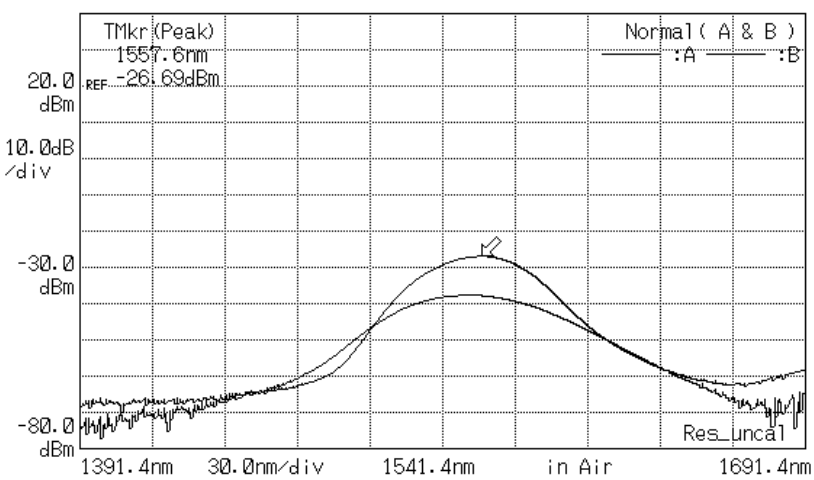

Figure 5. Comparison between the SOA and the HPS spectrum (the latter has a higher peak).
The fluctuation amplitudes are not high enough to generate true random numbers, therefore they are amplified by an EDFA. We apply a CWDM add-drop multiplexer as prefilter. The prefilter cuts off the unwanted sideband components, so that the EDFA does not amplify the whole band. It means that the accumulated energy is used to amplify in a narrower range, causing higher suppression of the EDFA's own noise. Here we use the CWDM standard, because it has a higher bandwidth than DWDM or other filters, so that the input power is large enough to turn on the EDFA. The filter has a bandwidth of $19.2 \mathrm{~nm}$, and the insertion loss is practically negligible. The filtered and unfiltered spectra are compared in Figure 6.

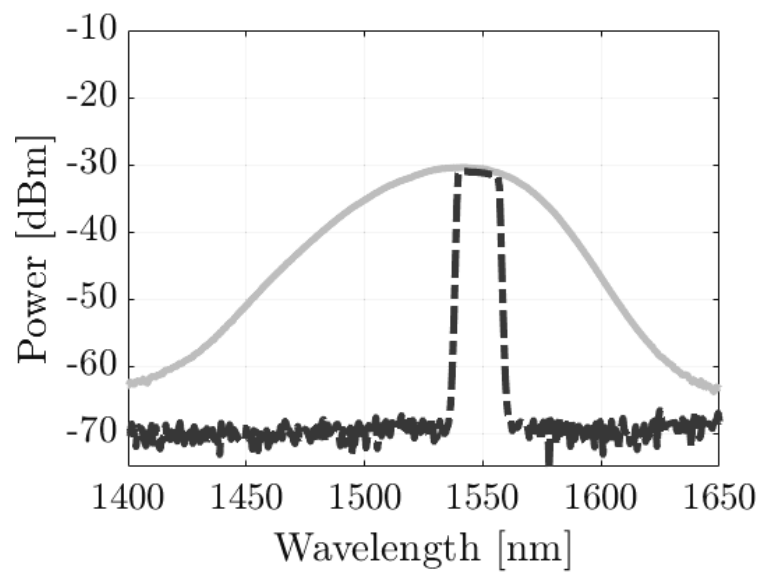

Figure 6. The unfiltered (cont.) and filtered (dashed) HPS spectrum.

The EDFA amplifies the signal significantly. The maximum of the optical power is at $1542.6 \mathrm{~nm}(-13.49 \mathrm{dBm})$. The EDFA's own noise is suppressed by $22.4 \mathrm{~dB}$. It is $7.4 \mathrm{~dB}$ higher compared to the case when the SOA is applied as the ASE source. However, the huge total power causes saturation in the optoelectrical converter. The EDFA amplifies everything within its gain spectrum, so using another filter at the end of the system is inevitable. We use a CWDM filter again, with the purpose of providing enough optical intensity that the gamma distribution converges to the symmetric normal distribution. This filter has $2.5 \mathrm{~dB}$ insertion loss around $1550 \mathrm{~nm}$. The output power after the second filter is $8.1 \mathrm{dBm}$, therefore we use an attenuator with around $3.5 \mathrm{~dB}$ attenuation. The detected power at the output of the optical system $(4.6 \mathrm{dBm})$ is high enough to avoid an asymmetric distribution, but low enough to avoid saturation. The amplified and filtered spectra are shown in Figure 7.

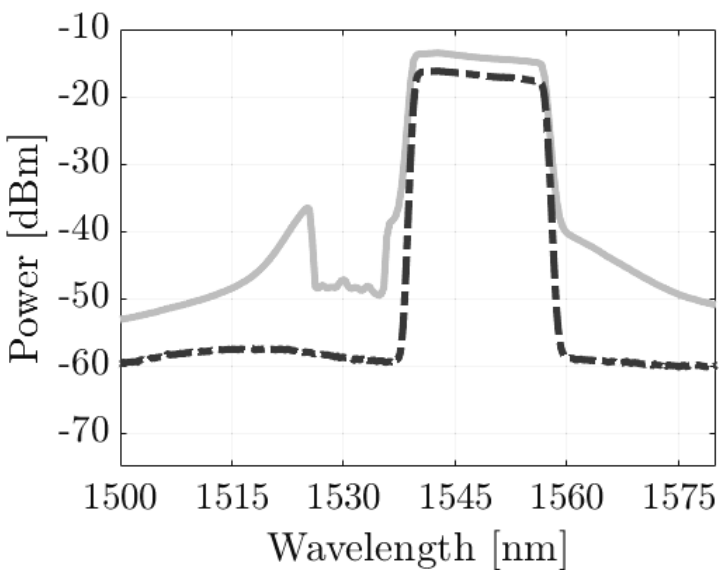

Figure 7. The HPS spectrum amplified by the EDFA (continuous) and the filtered optical spectrum after amplification (dashed). 
An optical-electrical converter (called a lightwave converter) is used to convert optical intensity to electrical voltage. The color scale display of the detected intensity-fluctuation is shown in Figure 8. It has an average of $631.87 \mathrm{mV}$ and peak-to-peak voltage of $250.07 \mathrm{mV}$. It is clearly visible that it is nearly symmetric, being beneficial in terms of randomness quality.

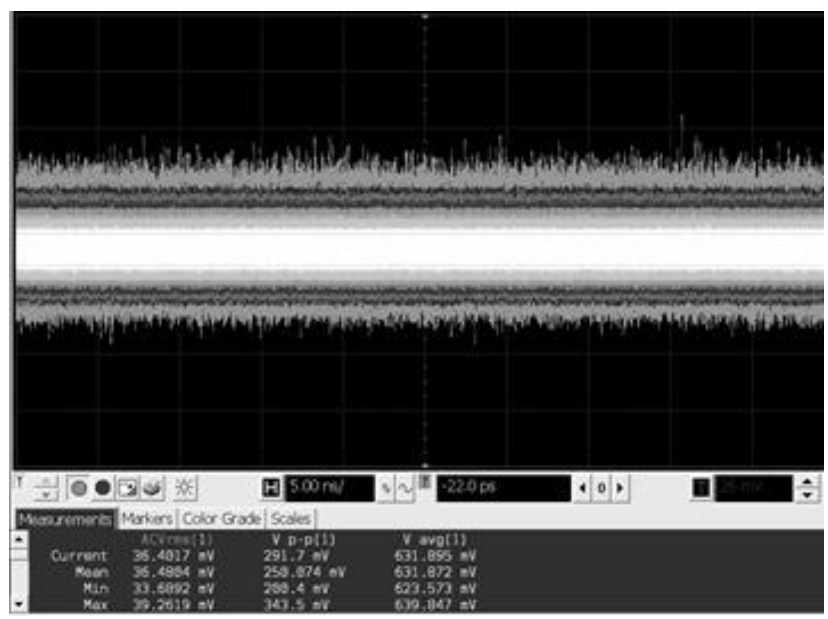

Figure 8 . The detected intensity-fluctuation.

\section{SAMPLING AND Post-Processing}

The electrical signal is digitized and stored by an oscilloscope and processed offline by Matlab [13]. The median compared samples provide theoretically uniformly distributed 0 and 1 bits. Due to the fact that we compared to the median, some values coincide with it. To not lose any bits, we added $50 \mu \mathrm{V}$ to these samples. It causes a deviation from the uniform distribution, but this intentional error highlights the differences between sampling rates. We assigned just one bit to the values, because the quality of random numbers in different rates shows higher contrast. The rates are chosen so that they cover a wide range around the analog bandwidth (the scope's $8 \mathrm{GHz}$ bandwidth is lower than the photoreceiver's, limiting the bit generation rate). The values were $0.1,0.2,0.5,1,2,4,10$ and $20 \mathrm{GSa} / \mathrm{s}$. Unfortunately, the sampling with $8 \mathrm{GSa} / \mathrm{s}$ was not supported by the oscilloscope. $10^{9}$ samples are collected for each sampling rate and 1000 bitstreams were created from these that consist of $10^{6}$ bits. All bit streams are then subjected to randomness testing. A decrease in the quality of randomness is expected with increasing sampling rate, because it causes short term correlations, especially for those higher than $8 \mathrm{GSa} / \mathrm{s}$. For the evaluation of randomness, we used the NIST (National Institute of Standards and Technology) [14] test suite that contains 15 different tests. All tests generate a p-value, representing the probability accepting the null hypothesis that the bitstream is random. This p-value needs to be higher than the significance level, and p-values should be uniformly distributed. Nevertheless, QRNGs should generate all sequences of a given length with the same probability. It means that the generator is expected to fail sometimes (at least once), but no more than 20 times out of 1000 sequences. This is the condition of the success. The results of testing are shown in Figure 9. There is a tendency as the sampling rate grows, decreasing the number of the successful tests (or keeping the value). There is just one exception: in case of $0.2 \mathrm{GSa} / \mathrm{s}$ there are more successful tests than in case of $0.1 \mathrm{GSa} / \mathrm{s}$.

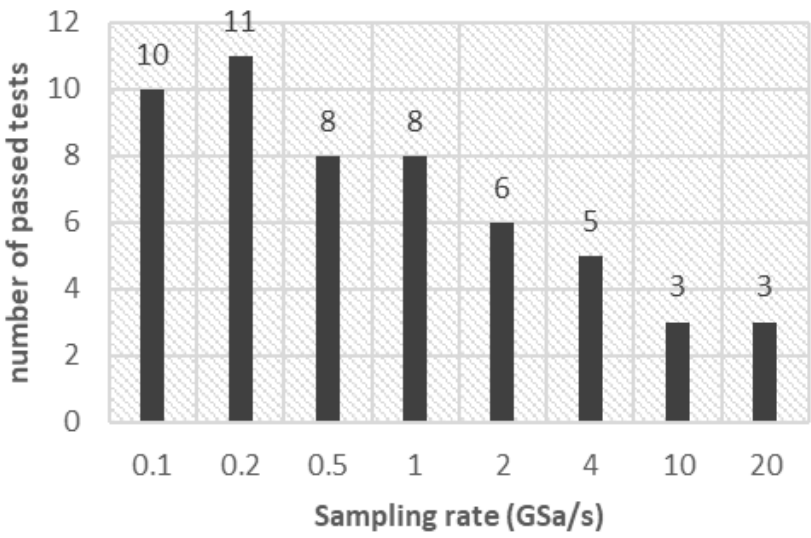

Figure 9. The number of passed NIST tests at different sampling rates.

In order to reduce correlations in the raw bit streams and increase the quality of randomness, we use a post-processing method. It was a simple XOR technique: we take the XOR operation of the original sequence with a delayed sequence (we apply a 20 bit delay). It drastically increased the number of passed tests, as it is clearly visible in Figure 10. This method reduced the short-term correlations. After post-processing, the bit sequences at $0.1 \mathrm{GSa} / \mathrm{s}, 0.5 \mathrm{GSa} / \mathrm{s}$ and $1 \mathrm{GSa} / \mathrm{s}$ passed everything. Two of them $(0.2 \mathrm{GSa} / \mathrm{s}$ and $4 \mathrm{GSa} / \mathrm{s})$ passed all except one test and one of them $(4 \mathrm{GSa} / \mathrm{s})$ passed all except two tests. The two sequences with sampling rates higher than the oscilloscope bandwidth $(8 \mathrm{GHz})$ also passed more tests than before the postprocessing, but despite of it, they are unsuitable for the single-bit generation in our system.

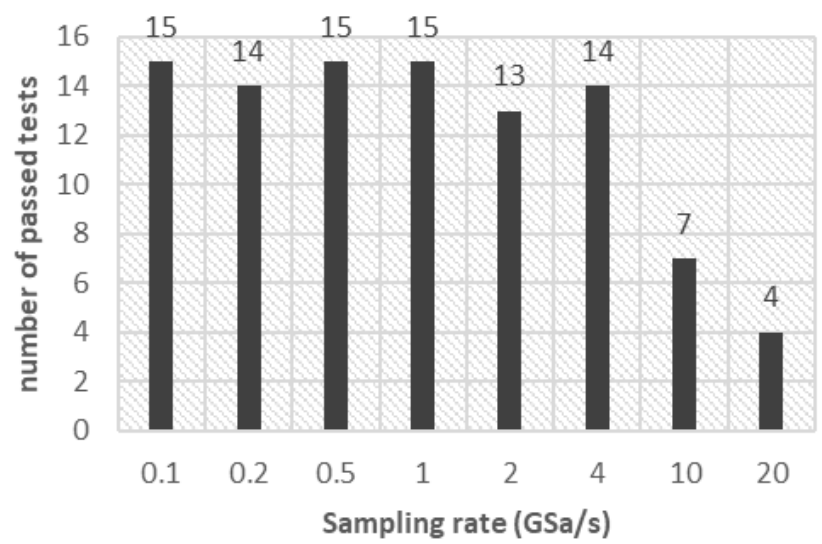

Figure 10. The number of passed NIST tests at different sampling rates after post-processing.

We can conclude that the randomness is heavily depending on the sampling rate. According to expectations, with the sampling rate being below the analog bandwidth, the quality of the randomness is better than above it. However, it means a decrease in the bit generation rate. It is foreseeable that the self-delayed XOR method can increase drastically the quality of randomness below the analog bandwidth. The optimal choice is $4 \mathrm{GSa} / \mathrm{s}$ for us, which is the highest sampling rate with just one unsuccessful test (suitable post-processing can eliminate this failure). There are some additional opportunities. Firstly, the mean will be a better comparison level than the median, because we can avoid the intentional comparison failure. Furthermore, we can increase the XOR delay, which could reduce the short-term correlations even more. Besides, we can assign more bits to one sample. Although it causes additional correlations, these can be reduced by discarding several MSBs. 
Amplified spontaneous emission based

quantum random number generator

\section{REAL-Time GENERATION}

As of now, our website is a trial version of the future online interface, which will operate with an electrical level shifting circuit, and it will be available for public use, but the current version is able to generate bits. This version only uses cheap equipment that limits the bit generation rate. The website runs on a Raspberry Pi. The Pi has no built-in ADC; the converteor runs on a distinct ESP8266 microcontroller. The hardware arrangement is visible in Figure 11.

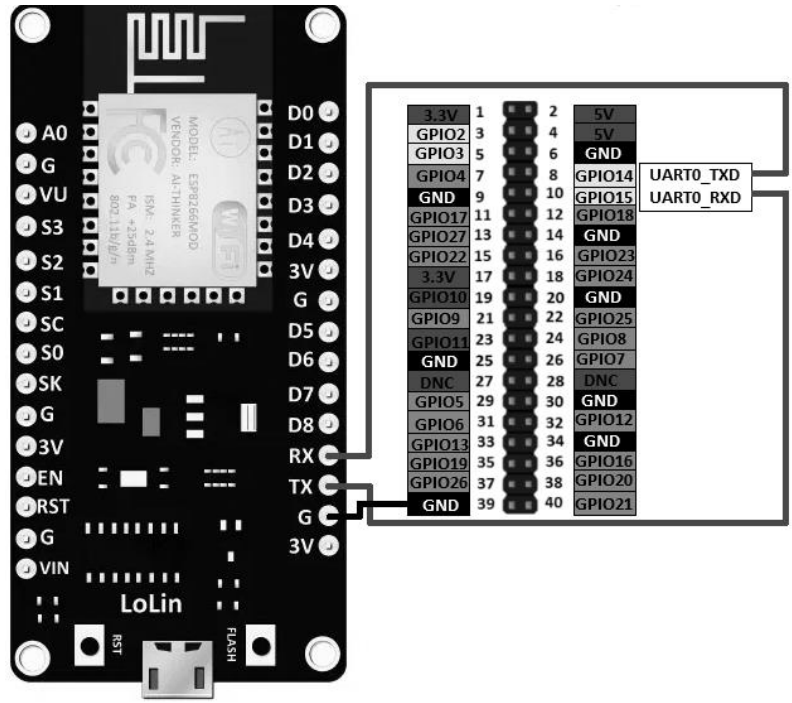

Figure 11. Block diagram of the real-time RNG.

The signal from the optical-electrical converter is sampled by a 10-bit ADC operating between $0 \mathrm{~V}$ and $3.3 \mathrm{~V}$. The communication between the microcontroller and the Pi is on UART.

The ESP8266 is programmed in C language. If the serial port becomes active, the equipment reads the number of the requested bits. Then the ESP8266 fills an n-bit array from the analog input. The equipment repeats this method every time the Pi requests bits, so the reuse of samples is excluded. These values are between 0 and 1024 . We compare the values to the mean and refill the n-bit array with 0 and 1 bits (bigger $\mathrm{n}$ means more precise average). Then the equipment creates a string with suitable size (it is equal to the number of the requested bits) and this string is transmitted to the $\mathrm{Pi}$ on a serial port.

The Raspberry Pi is programmed in HTML, CSS, PHP, Python and SQL languages. The website is created by a PHP file, where the number of bits to receive can be specified. The "Generate" button creates an SQL record, which requests bits from the ESP8266. The requesting, processing and ready state have different flags in the SQL table. Then the .php file starts the.$p y$ file. This file selects the record (which is in a sending state) from the SQL table and requests the number of the requested bits. This number is sent to the ESP8266 by the .py file, and while the microcontroller sends back the appropriate amount of bits, the file creates a SQL record in processing state. Finally, the .php file writes the bits onto the webpage.

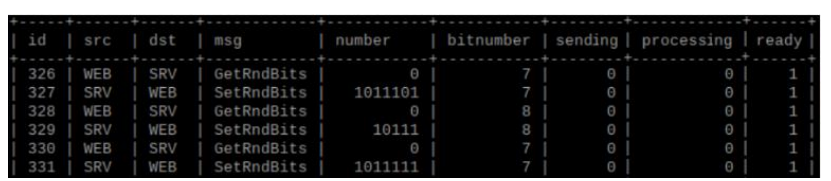

Figure 12. The registration system.
There are some additional opportunities to develope. Currently a level shifting circuit is in the development phase. This circuit removes the mean from the measured intensity-fluctuation, then extends the fluctuation levels to the whole range of the ADC with a multiplier circuit and offset voltage. The advantage of this circuit is that there would be no need to calculate the average, because the system provides bits, where the comparison threshold is the half-range of the ADC. Another opportunity is to operate the real-time bit generating system as a real webserver. Currently the system just operates on a local network, but the access is expansible for everyone. Finally, higher bitgeneration rates are achievable with sampling for instance on 16-32 bits. By discarding several MSBs, the problems of oversampling are reducible.

\section{CONCLUSION}

The QRNG presented in this paper is based on ASE, a truly random quantum mechanical process. The ASE noise is filtered with the drop channel of a CWDM add-drop multiplexer. An EDFA is used to amplify this signal to avoid the asymmetry of the intensity distribution. After the EDFA, a second CWDM filter (and an attenuator) is applied to reduce the power in order to avoid the saturation of the receiver. After the optical-electrical converter, the intensity-fluctuation was single-bit sampled offline with Matlab. The effects of sampling rate were tested, and the conclusion was that below $8 \mathrm{GSa} / \mathrm{s}$ (the analog bandwidth of oscilloscope) the sampling rate is acceptable to generate random bit sequences. However, the application of post-processing is inevitable to increase the quality of randomness. The optimal sampling rate is $4 \mathrm{GSa} / \mathrm{s}$ with a suitable post-processing method, because it is the highest available bit generation rate below $8 \mathrm{GSa} / \mathrm{s}$. Furthermore, the developed webserver running on a Raspberry $\mathrm{Pi}$ provides the opportunity to generate bits real-time with a registry system. Further improvements might be done to the system, so that the bit generation can be effectively reduced to a zero-level comparison, and the realtime bit generation rate can be increased to be closer to the what could be theoretically possible.

\section{ACKNOWLEDGMENT}

The research has been supported by the European Union, co-financed by the European Social Fund (EFOP-3.6.2-162017-00013, Thematic Fundamental Research Collaborations Grounding Innovation in Informatics and Infocommunications).

\section{REFERENCES}

[1] L. Li, A. Wang, P. Li, H. Xu, L. Wang, and Y. Wang, „Random bit generator using delayed self-difference of filtered amplified spontaneous emission,” IEEE Photonics Journal, vol. 6, no. 1, pp. 1-9, 2014.

[2] A. Argyris, E. Pikasis, S. Deligiannidis, and D. Syvridis, „Sub-tb/s physical random bit generators based on direct detection of amplified spontaneous emission signals," Journal of Lightwave Technology, vol. 30, no. 9, pp. 1329-1334, 2012.

[3] Y. Liu, M. Zhu, B. Luo, J. Zhang, and H. Guo, ,Implementation of 1.6 Tbs-1 truly random number generation based on a super-Á. Marosits et al.: Amplified spontaneous emission based quantum random number generator luminescent emitting diode,'Laser Physics Letters,vol.10, no. 4, p. 045001, 2013.

[4] C. R. Williams et al., "Fast physical random number generator using amplified spontaneous emission," Optics express, vol. 18, no. 23, pp. 23584-23597, 2010

[5] X. Li et al., "Scalable parallel physical random number generator based on a superluminescent led," Optics letters, vol. 36, no. 6, pp. 1020-1022, 2011. 
[6] R. Paschotta, article on 'stimulated emission' in the Encyclopedia of Laser Physics and Technology, 1. edition October 2008, Wiley-VCH, ISBN 978-3-527-40828-3

[7] R. Paschotta, article on 'spontaneous emission' in the Encyclopedia of Laser Physics and Technology, 1. edition October 2008, Wiley-VCH, ISBN 978-3-527-40828-3

[8] R. Paschotta, article on 'amplified spontaneous emission' in the Encyclopedia of Laser Physics and Technology, 1. edition October 2008, Wiley-VCH, ISBN 978-3-527-40828-3

[9] M. Herrero-Collantes and J. C. García-Escartín, "Quantum random number generators," Reviews of Modern Physics, vol. 89, no. 1, p. 015004, 2017.

[10] R. Paschotta, article on 'semiconductor optical amplifiers' in the Encyclopedia of Laser Physics and Technology, 1. edition October 2008, Wiley-VCH, ISBN 978-3-527-40828-3

[11] R. Paschotta, article on 'erbium-doped fiber amplifiers' in the Encyclopedia of Laser Physics and Technology, 1. edition October 2008, Wiley-VCH, ISBN 978-3-527-40828-3

[12] R. Paschotta, article on 'four-level and three-level gain media' in the Encyclopedia of Laser Physics and Technology, 1. edition October 2008, Wiley-VCH, ISBN 978-3-527-40828-3

[13] Á. Schranz, Á. Marosits, and E. Udvary, „Effects of sampling rate on amplified spontaneous emission based single-bit quantum random number generation," in 2019 21st International Conference on Transparent Optical Networks (ICTON), pp. 1-4, IEEE, 2019.

[14] A. L. Rukhin et al., „A statistical test suite for random and pseudorandom number generators for cryptographic applications," tech. rep., National Institute of Standards \& Technology, Gaithersburg, MD, United States, 2010. Spec. Pub. 800-22, Rev. 1a

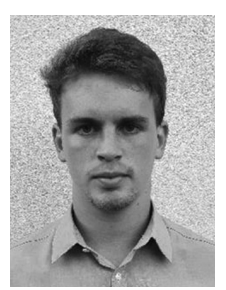

Ádám Marosits is currently a BSc student in electrical engineering at the Budapest University of Technology and Economics (BME, Budapest, Hungary) at the Department of Broadband Infocommunications and Electromagnetic Theory. He is a member of the Balatonfüred Student Research Group. His research interests include quantum communication and quantum random number generation, furthermore the optimization with quantum annealing.

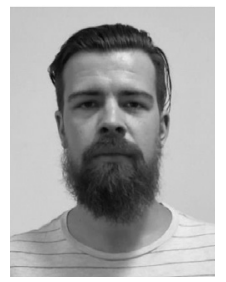

Ágoston Schranz received his BS and MS degrees in electrical engineering from the Budapest University of Technology and Economics (BME), Budapest, Hungary, in 2015 and 2017, respectively. Currently, he is working toward his $\mathrm{PhD}$ in electrical engineering at the Department of Broadband Infocommunications and Electromagnetic Theory. He is a member of the Optical and Microwave Telecommunication Laboratory and the Balatonfüredi Student Research Group. His research interests include optical communications, quantum key distribution, and quantum random number generation.

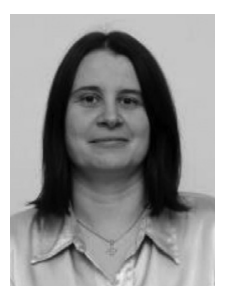

Eszter Udvary (M'98) received the $\mathrm{PhD}$ degree in electrical engineering from Budapest University of Technology and Economics (BME), Budapest, Hungary, in 2009. She is currently an Associate Professor at BME, Department of Broadband Infocommunications and Electromagnetic Theory, where she leads the Optical and Microwave Telecommunication Lab Dr Udvary's research interests are in the broad areas of optical communications, include optical and microwave communication systems, Radio over fibre systems, optical and microwave interactions and applications of individual electrooptical devices. Her current research interests are future quantum and visible light communications. 